\title{
K. Metalloproteins
}

\section{K-01}

\section{Recent advances in urease metallobiochemistry}

Robert P. Hausinger

Michigan State University, East Lansing, MI, USA.

hausinge@msu.edu

Klebsiella aerogenes urease possesses three dinuclear nickel active sites embedded in a (Ure ABC $)_{3}$ structure. Synthesis of this deeply buried metallocenter requires UreD, UreF, and UreG, possibly acting in concert as a GTP-dependent molecular chaperone, with UreE serving as a metallochaperone. Computational and chemical cross-linking evidence suggests that UreB moves in a hinge-like motion to allow access to the nascent active site. To explore this hypothesis, (UreAC) ${ }_{3}$ and UreB were separately isolated and examined. UreD binds to UreB in the Ure ABC-UreD complex of urease apoprotein according to small-angle X-ray scattering and cross-linking studies, and a soluble functional form of UreD was generated and studied in isolation and in various complexes. Soluble and functional forms of UreF were studied alone and in the UreABC-UreDF complex. The metal-binding properties of purified UreG and its mutants were investigated. The effects of the UreG mutations on urease activation were also discerned. Furthermore, UreABC-UreDFG and other complexes were studied. In addition to this effort focused on elucidating the $K$. aerogenes urease maturation process, evidence related to a naturally occurring nonnickel urease in Helicobacter mustelae will be presented.

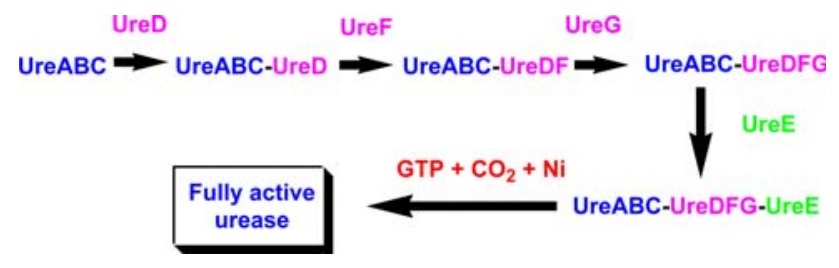

K-02

Unraveling the catalytic mechanism of nitrile hydratases

Richard C. Holz, Saroja Rao, Sanghamitra Mitra, Ashley Freeman

Department of Chemistry, Loyola University-Chicago, 1068 West Sheridan Road, Chicago, IL 60626, USA. Rholz1@luc.edu

Nitrile hydratase (NHase, EC 4.2.1.84) catalyzes the hydration of nitriles to their corresponding higher value amides in a chemo-, region-, and/or enantio-selective manner at ambient pressures and temperatures at physiological $\mathrm{pH}$ [1]. NHases contain either a nonheme $\mathrm{Fe}$ (III) ion (Fe-type) or a non-corrin $\mathrm{Co}(\mathrm{III})$ ion (Co-type) in their active site [2]. X-ray crystal structures of both Co-and Fe-type enzymes have been solved and reveal that the M(III) center is six coordinate with the remaining ligands made up of three cysteines, and two amide nitrogens (Fig. 1). Two of the active site cysteine residues are post-translationally modified to cysteine-sulfinic acid $\left(-\mathrm{SO}_{2} \mathrm{H}\right)$ and cysteine-sulfenic acid (-SOH) yielding an unusual metal coordination geometry, which was termed a "claw-setting". In order to gain insight into the catalytic mechanism of NHase enzymes, we have kinetically and spectroscopically analyzed the Co-type NHase from

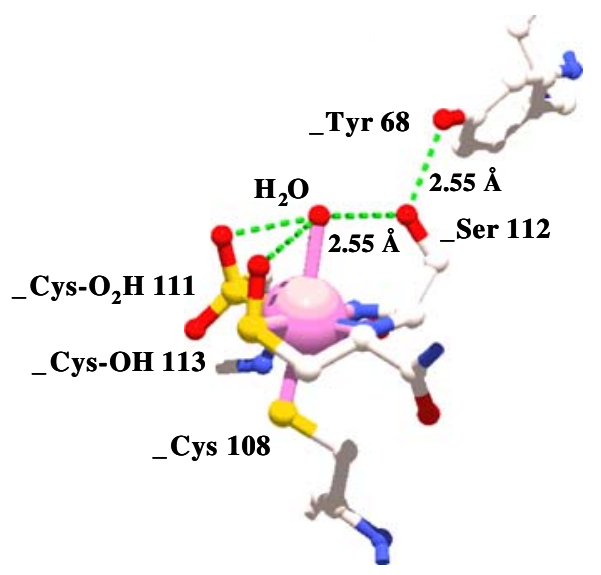

Fig. 1 Active site of NHase from P. thermophilia (1IRE)

Pseudonocardia thermophila JCM 3095 (PtNHase) [3] and the Fetype NHase from Comamonas testosteroni Nil (CtNHase) [4]. Combination of these data with those previously reported for NHase enzymes and model complexes has allowed a novel catalytic mechanism for NHase enzymes to be proposed.

\section{References}

1. Banerjee A, Sharma R, Banerjee UC (2002) The nitrile-degrading enzymes: current status and future prospects. Appl Microbiol Biotechnol 60:33-44

2. Kovacs JA (2004) Synthetic analogues of cysteinate-ligated nonheme iron and non-corrinoid cobalt enzymes. Chem Rev 104:825-848 3. Mitra S, Holz RC (2007) Unraveling the catalytic mechanism of nitrile hydratases. J Biol Chem 282:7397-7404

4. Rao SN, Holz RC (2008) Analyzing the catalytic mechanism of the Fe-type nitrile hydratase from Comamonas testosteroni Ni1. Biochemistry 47:12057-12064

\section{K-03}

\section{Biochemistry of the Ni(II)-dependent glyoxalase I} enzymes

\section{John Honek ${ }^{1}$}

${ }^{1}$ Department of Chemistry, University of Waterloo, Waterloo, ON N2L 3G1, Canada, jhonek@uwaterloo.ca

Glyoxalase I (GlxI) is the first of two enzymes in the two-component glyoxalase system that functions to remove cellular cytotoxic $\alpha$-ketoaldehydes. The metalloenzyme GlxI catalyzes the isomerization of the non-enzymatically formed hemithioacetal of methylglyoxal and glutathione (GSH) to $S$-D-lactoylglutathione. Glyoxalase II hydrolyses the product of the GlxI reaction to D-lactate and free GSH. Recent investigations have shown that there are two classes of metal-activated GlxI enzymes: a $\mathrm{Zn}^{2+}$-activated class and a non- $\mathrm{Zn}^{2+}$-activated (but $\mathrm{Ni}^{2+} / \mathrm{Co}^{2+}$-activated) class. Our recent findings in this area will be presented (Fig. 1). 


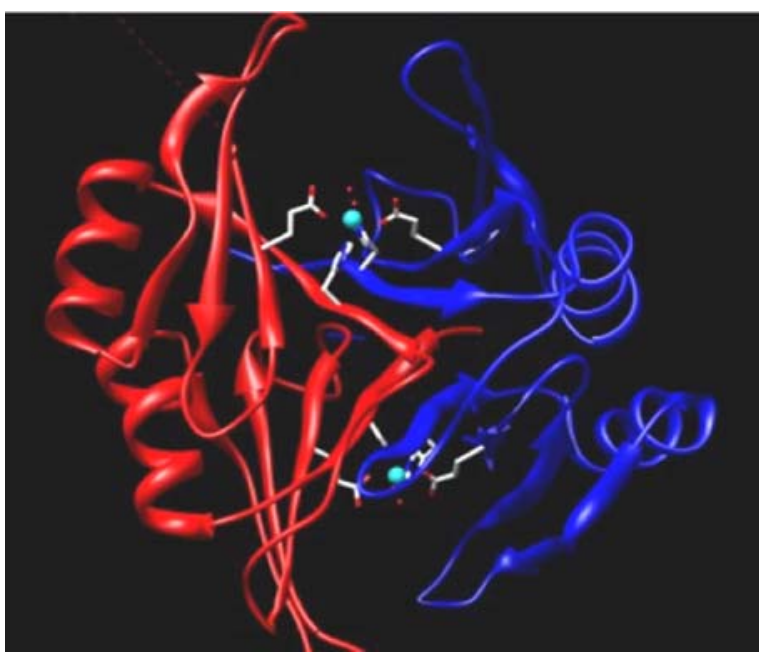

Fig. 1 E. coli $\mathrm{GlxI}$-Ni(II) form

\section{Reference}

1. Sukdeo N, Daub E, Honek JF (2007) Biochemistry of the nickeldependent glyoxalase I enzymes. In: Sigel A, Sigel H, Sigel RKO (eds) Metal ions in life sciences, vol 2. Wiley, New York, pp 445-472.

\section{K-04}

\section{Metal-dependent nonoxidative decarboxylation reaction} Aimin Liu

Department of Chemistry, Georgia State University, Atlanta, GA 30303, USA. feradical@gsu.edu

Decarboxylases typically utilize organic cofactor and acid-base chemistry, or a transition metal coupled with dioxygen to activate their substrates. Recently, a nonoxidative decarboxylation catalyzed by an obligatory transition metal cofactor has been characterized. The enzyme $\alpha$-amino- $\beta$-carboxymuconate- $\varepsilon$-semialdehyde decarboxylase (ACMSD) utilizes a mononuclear metal ion to decarboxylate the substrate in an oxidant-independent manner. Biochemical and structural characterizations of this enzyme have confirmed that ACMSD is a member of the amidohydrolase superfamily. Further studies have established that ACMSD is a prototype member of a new subfamily within the superfamily that catalyzes metal-dependent $\mathrm{C}-\mathrm{C}$ bond cleavages. The catalytic mechanism, structural features, evolution, and biological significance of this ACMSD and the homologues of the new amidohydrolase- 2 superfamily will be discussed.

\section{Reference}

1. Liu A, Zhang H (2006) Biochemistry 45:10407-10411

\section{K-05}

Metal ion mutagenesis: conversion of a purple acid phosphatase from sweet potato to a neutral phosphatase with the formation of an unprecedented catalytically competent $\mathrm{Mn}^{\text {II }} \mathrm{Mn}^{\text {II }}$ active site Nataša Mitić ${ }^{1}$, Christopher J. Noble ${ }^{2}$, Lawrence R. Gahan ${ }^{1}$, Graeme R. Hanson ${ }^{1}$, Gerhard Schenk ${ }^{2}$

${ }^{1}$ School of Chemistry and Molecular Biosciences, The University of Queensland, Brisbane Qld 4072, Australia.

${ }^{2}$ Centre of Magnetic Resonance, The University of Queensland, Brisbane Qld 4072, Australia. graeme.hanson@cmr.uq.edu.au Purple acid phosphatases (PAPs) require a heterovalent binuclear metal ion center for catalysis under acidic conditions. Sweet potato PAP is unusual in that it appears to have a specific requirement for manganese, forming a unique $\mathrm{Fe}^{\mathrm{III}}-\mu-(\mathrm{O})-\mathrm{Mn}^{\mathrm{II}}$ center under catalytically optimal conditions. Herein we demonstrate, with kinetic and detailed EPR spectroscopic studies, that in this enzyme the chromophoric Fe ${ }^{\mathrm{III}}$ can be replaced by $\mathrm{Mn}^{\mathrm{II}}$, forming a catalytically active, unprecedented antiferromagnetically coupled homodivalent $\mathrm{Mn}^{\mathrm{II}}-\mu-(\mathrm{H}) \mathrm{OH}-\mu$-carboxylato$\mathrm{Mn}^{\mathrm{II}}$ center in a PAP. However, while the enzyme is still purple, it no longer functions as an acid phosphatase, having optimal activity at neutral $\mathrm{pH}$. Thus, PAPs may have evolved from distantly related divalent binuclear metallohydrolases that operate under $\mathrm{pH}$ neutral conditions, by stabilization of a trivalent-divalent metal ion core. The present $\mathrm{Mn}^{\mathrm{II}}-\mathrm{Mn}^{\mathrm{II}}$ system models these distant relatives, and the results herein make a significant contribution to our understanding of the role of the chromophoric metal ion as an activator of the nucleophile.

\section{Reference}

1. Schenk G, Gahan LR, Carrington LE, Mitić N, Valizadeh M, Hamilton SE, de Jersey J, Guddat LW Proc Natl Acad Sci 102:273-278

\section{K-06}

Spectroscopic and electronic structure studies probing the reaction coordinate of molybdenum hydroxylases Martin L. Kirk ${ }^{1}$, Abebe Habtegabre ${ }^{1}$, Joseph Sempombe ${ }^{1}$, Dingguo $\mathrm{Xu}^{1}$, Hua Guo ${ }^{1}$, Sushilla Z. Knottenbelt ${ }^{1}$

${ }^{1}$ Department of Chemistry and Chemical Biology, The University of New Mexico, MSC03 2060, 1 University of New Mexico, Albuquerque, NM 87131-0001. mkirk@unm.edu

The pyranopterin molybdenum enzymes function to catalyze various two-electron redox reactions coupled to formal oxygen atom transfer. They are ubiquitous in nature with eukaryotic, prokaryotic, and archaeal representatives and catalyze a wide variety of important reactions in the metabolism of carbon, sulfur, and nitrogen compounds. Xanthine oxidase (XO) is the prototypical Mo hydroxylase. $\mathrm{XO}$ oxidizes a variety of aldehydes and heterocyclic compounds, and both XO and the related aldehyde oxidase have been implicated in prodrug activation and drug metabolism. We have shown that the nature of the redox active molecular orbital has a profound influence on the MoOS bonding scheme and that the redox state of MoOS systems directly affects the relative electro-/nucleophilicity of the terminal sulfido donor [1]. We have undertaken a detailed spectroscopic and electronic structure study of XO in order to understand the key electronic structure contributions to reactivity at the active site. Here, we present new results that provide detailed insight into the nature of the reaction coordinate during the reductive half-reaction of $\mathrm{XO}$ [2]. The key results here provide new information regarding the formal hydride transfer process that results in substrate oxidation and Mo reduction.

\section{References}

1. Doonan CJ, Rubie RD, Peariso K, Harris HH, Knottenbelt SZ, George GN, Young CG, Kirk ML (2008) J Am Chem Soc 130:55-65 2. Kirk ML, Knottenbelt SZ, Habtegabre A (2009) Comput Inorg Bioinorg Chem (accepted for publication)

\section{K-7}

\section{Nature and role of molybdopterin in the function} of oxotransferase enzymes

C. David Garner, Freya Hine, E. Stephen Davies, Jon McMaster School of Chemistry, The University of Nottingham, University Park, Nottingham, NG7 2RD, UK. dave.garner@nottingham.ac.uk Each molybdenum oxotransferase enzyme catalyses a reaction, the net effect of which is the transfer of an oxygen atom either to or from the substrate: $\mathrm{X}+\mathrm{H}_{2} \mathrm{O} \leftrightarrow \mathrm{XO}+2 \mathrm{H}^{+}+2 \mathrm{e}^{-}$. The catalysed 
reactions involve a wide range of substrates, including $\mathrm{SO}_{3}{ }^{2-}$ to $\mathrm{SO}_{4}{ }^{2-}$ by the sulfite oxidases, $\mathrm{NO}_{3}{ }^{-}$to $\mathrm{NO}_{2}{ }^{-}$by the nitrate reductase and DMSO to DMS by the DMSO reductase. The nature of many of the molybdenum oxotransferase enzymes is known in considerable detail, thanks to an extensive range of biochemical studies and X-ray protein crystallographic characterization. In each case, the catalytic centre involves molybdenum bonded to one or two 'molybdopterin' (MPT) cofactors, the nature of which can be represented as:<smiles>CSC1=C([S-])C2Nc3c(nc(N)[nH]c3=O)NC2OC1COP(O)O</smiles>

We have attempted to synthesize MPT and close chemical relatives to investigate the chemical properties of these systems, notably their redox character. Also, we have performed DFT calculations to probe the electronic structure of various forms of MPT. Our aim has been to improve the understanding of the role(s) of MPT in the catalyses effected by the molybdenum oxotransferase enzymes.

\section{K-08}

\section{Arsenic metalation pathways in metallothioneins}

\section{Thanh T. Ngu, Martin J. Stillman}

Department of Chemistry, The University of Western Ontario, London, ON N6A 5B7, Canada. Martin.Stillman@uwo.ca

Mammalian metallothionein is a small, cysteine-rich, multiple metalbinding protein. When bound to divalent metals, mammalian metallothionein forms $\mathrm{M}_{3} \mathrm{Cys}_{9}(\beta)$ and $\mathrm{M}_{4} \mathrm{Cys}_{11}(\alpha)$ metal-thiolate clusters in two domains. This paper describes in detail arsenic-metalation of metallothionein and the metal-induced folding pathway mechanism for metallothionein using time-resolved and temperature-resolved electrospray ionization mass spectrometry to probe metal binding in real time. We report the first complete metalation pathway for any metallothionein. Comprehensive kinetic parameters for the individual fragments, and the complete two-domain proteins from human and the brown seaweed Fucus vesiculosus are described. The evolutionary advantage for the two-domain structure of metallothionein will be demonstrated. The significance on metal binding of linker length between the domains will be described. Molecular models for arsenicmetallothionein have been calculated.
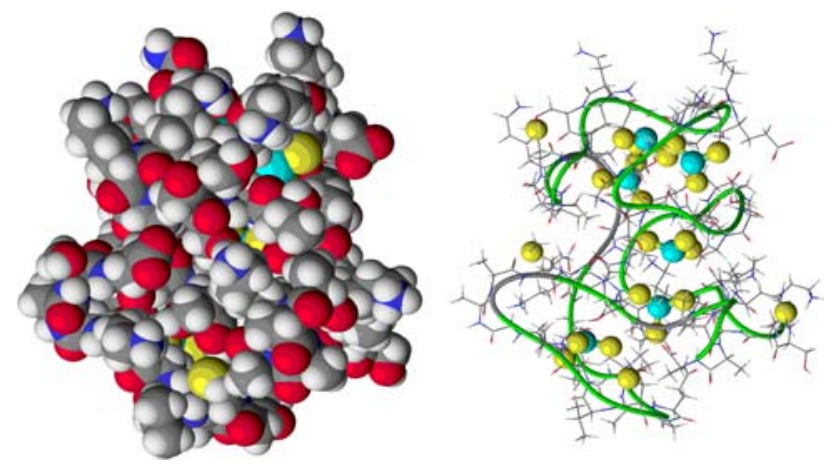

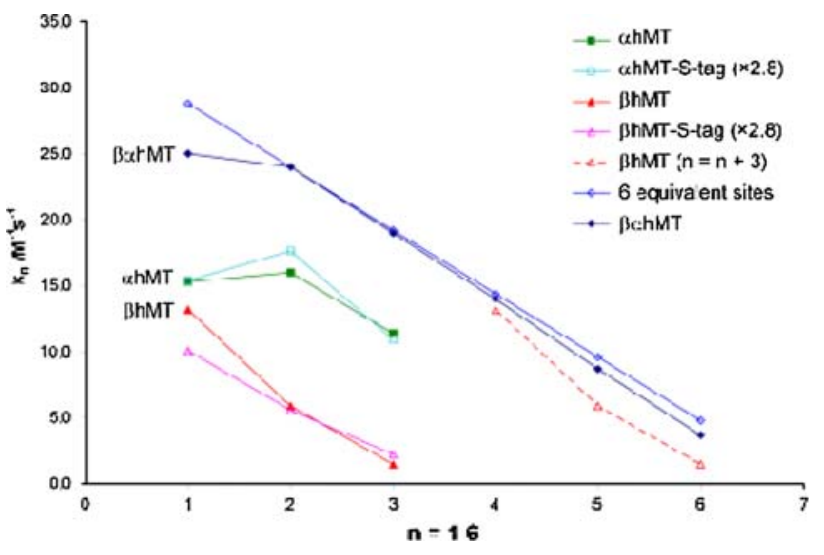

References

1. Ngu T, Easton A, Stillman MJ (2008) J Am Chem Soc 130:1701617028

2. Ngu TT, Stillman MJ (2006) J Am Chem Soc 128:12473-12483

3. Ngu TT, Stillman MJ (2009) IUBMB Life (in press)

\section{K-09}

Structures of the $M o(V)$ centers of sulfite oxidizing enzymes from variable frequency pulsed epr spectroscopy and DFT calculations for "difficult" nuclei John H. Enemark ${ }^{1}$, Arnold M. Raitsimring ${ }^{1}$, Andrei V. Astashkin ${ }^{1}$, Eric L. Klein ${ }^{1}$, Kayunta Johnson-Winters ${ }^{1}$, Alexei Potapov ${ }^{2}$, Daniella Goldfarb ${ }^{2}$, Dmitry Ganyushin ${ }^{3}$, Christoph Riplinger ${ }^{3}$, Frank Neese ${ }^{3}$

${ }^{1}$ Department of Chemistry and Biochemistry, University of Arizona, Tucson, AZ 85721-0041, USA.

${ }^{2}$ Department of Chemical Physics, Weizmann Institute of Science, 76100 Rehovot, Israel.

${ }^{3}$ Institut für Physikalische und Theoretische Chemie, Universität Bonn, Wegelerstrasse 12, 53115 Bonn, Germany.

jenemark@u.arizona.edu

Sulfite-oxidizing enzymes (SOEs) occur in all forms of life. During catalysis the five-coordinate square pyramidal oxo-Mo active site passes through the $\mathrm{Mo}(\mathrm{V})$ state, and intimate details of the structure can be obtained from variable frequency pulsed EPR spectroscopy through the hyperfine interactions $(h f i)$ and nuclear quadrupole interactions (nqi) of nearby magnetic nuclei. This study focuses on ${ }^{17} \mathrm{O}$, ${ }^{33} \mathrm{~S}$, and ${ }^{35,37} \mathrm{Cl}$; these nuclei are "difficult" to detect and have been barely investigated by pulsed EPR methods. Optimization of the spectrometer operational frequencies and the measurement conditions allows detection of these "difficult" nuclei and simplifies the interpretation of the spectra. Studies of isotopically labeled model $\mathrm{Mo}(\mathrm{V})$ compounds and quantum-chemical calculations also facilitate the interpretation of spectroscopic data from SOEs. Recent results include: (1) the detection of bound sulfate for several mutants of SOEs using ${ }^{33} \mathrm{~S}(I=3 / 2)$ labeling, (2) unambiguous direct detection of ${ }^{35,37} \mathrm{Cl}^{-}$ $(I=3 / 2)$ in close proximity to the $\mathrm{Mo}(\mathrm{V})$ center of the low $\mathrm{pH}$ form of $\mathrm{SO}$ and demonstration by DFT calculations that $\mathrm{Cl}^{-}$is not directly coordinated to the $\mathrm{Mo}(\mathrm{V})$ center, but rather associated through hydrogen-bonding to the $\mathrm{Mo}-\mathrm{OH}$ group in the equatorial plane, (3) labeling of SOEs with ${ }^{17} \mathrm{O}(I=5 / 2)$ and using electron spin echo envelope modulation (ESEEM) methods at $K_{\mathrm{a}}$-band $(\sim 30 \mathrm{GHz})$ and $\mathrm{W}$-band $(95 \mathrm{GHz})$ to directly determine the $h f i$ and $n q i$ of $\mathrm{Mo} \equiv{ }^{17} \mathrm{O}$ and $\mathrm{Mo}-{ }^{17} \mathrm{OH}$ groups as structural probes of the active sites of SOEs. 


\section{K-10}

\section{Mechanisms of hydroxylation of xanthine oxidoreducatase}

\section{Takeshi Nishino}

Department of Biochemistry and Molecular Biology, Nippon Medical School, Tokyo 113-8602, Japan. nishino@nms.ac.jp

Xanthine oxidoredactase (XOR) is a molybdenum containing flavoprotein found from bacteria to humans. The enzyme is a homodimeric protein of $\mathrm{Mr} \mathrm{300,000} \mathrm{and} \mathrm{is} \mathrm{composed} \mathrm{of} \mathrm{independent} \mathrm{subunits;} \mathrm{each}$ subunit contains one molybdopterin, two non-identical iron sulfur centers ([2Fe-2S] clusters) and one FAD. The oxidative hydroxylation of hypoxanthine, xanthine or various heterocyclic compounds takes place at the molybdenum center, and electrons thus introduced are transferred rapidly via two iron sulfur centers to FAD, where physiological oxidation by $\mathrm{NAD}^{+}$or molecular oxygen occurs [1]. In oxidized enzyme, the metal is in the $\mathrm{Mo}(\mathrm{VI})$ oxidation state, bearing an oxo- $(=\mathrm{O})$, one hydroxo $(\mathrm{OH})$ and a sulfido ligand. In the course of turnover reaction, the oxygen atom at the catalytically labile site $(\mathrm{OH})$ on the Mo center is transferred to a substrate and subsequently the labile site is regenerated by oxygen derived from the solvent. In this paper, the mechanism of substrate activation and roles of the amino acids' residues in the active site will be discussed based on the crystal structures of bovine milk and rat enzymes bound with various compounds as well as the results from site directed mutagenesis experiments, where the important residues in the active site were replaced by other amino acids.

\section{Reference}

1. Nishino T, Pai EF (2000) In: Messerschmidt A (ed) Xanthine oxidoreductase in handbook of metalloproteins. Wiley, New York, pp 1-14

\section{K-11}

Molecular basis for sulfite oxidation: what we can learn from bacterial enzymes

\section{Ulrike Kappler ${ }^{1}$, Trevor D. Rapson ${ }^{1}$, Jeremy Wilson ${ }^{1}$,} Paul V. Bernhardt ${ }^{1}$

${ }^{1}$ Centre for Metals in Biology, School of Chemistry and Molecular Biosciences, The University of Queensland, St. Lucia, Australia. u.kappler@uq.edu.au

Sulfite-oxidizing enzymes (SOEs) are evolutionarily ancient molybdoenzymes that are found in all forms of life where they contribute to the detoxification of sulfite. Despite the early discovery of the vertebrate variants of these enzymes, many aspects of the catalytic properties of SOEs are still unclear, including the mechanisms and pathways for intramolecular electron transfer (IET), and the implications of small changes to the active site environment for the catalytic competency of the enzymes.

Within this enzyme family, bacterial SOEs are the biggest and most varied group, and their structural characteristics make them excellent models for investigations of sulfite oxidation. Using bacterial SOEs as models we have been able to show that the amino acid environment of the active site is crucial in shaping enzymatic activity. A central arginine residue is critical for substrate binding, but at the same time adversely influences IET between the molybdenum active site and the accessory heme redox centre, while a histidine residue is responsible for maintaining substrate affinity at low $\mathrm{pH}$ [1]. As a result of our work it has become apparent that in contrast to previous models several possible pathways exist for the early events of enzymatic sulfite oxidation [2].

\section{References}

1. Bailey S, Rapson T, Winters-Johnson K et al (2009) J Biol Chem 284:2053-2063
2. Emesh S, Rapson TD, Rajapakshe A et al (2009) Biochemistry (in press)

\section{K-12}

Molybdenum induces the expression of a new Mo-Fe heterometallic protein in Desulfovibrio alaskensis Marta S.P. Carepo ${ }^{1}$, Maria G. Rivas ${ }^{1}$, Cristiano S. Mota ${ }^{1}$, Malgorzata Korbas $^{2}$, Marie-Claire Durand ${ }^{3}$, Ana T. Lopes ${ }^{1}$, Sandra C.M. Matos ${ }^{1}$, Carlos D. Brondino ${ }^{4}$, Alice S. Pereira ${ }^{1}$, Graham N. George ${ }^{2}$, Alain Dolla ${ }^{3}$, José J. G. Moura ${ }^{1}$, Isabel Moura ${ }^{1}$

${ }^{1}$ REQUIMTE/CQFB, Departamento de Química, FCT-Universidade nova de Lisboa, Caparica, Portugal.

${ }^{2}$ Department of Anatomy and Cell Biology, University of

Saskatchewan, Saskatoon, Canada.

${ }^{3}$ Unité Interactions et Modulateurs de Réponses, IBSM-CNRS, Marseille, France.

${ }^{4}$ Physics Department, FBCB, Universidad Nacional del Litoral, Santa Fe, Argentina. marta.carepo@dq.fct.unl.pt

Sulfate-reducing bacteria (SRB) contain several Mo-proteins that have been characterized; however, there are no reports addressing Mo homeostasis. In this work we report the characterization of a novel Mo$\mathrm{Fe}$ protein (MorP) associated with a system that responds to Mo in Desulfovibrio alaskensis. MorP is a periplasmic homomultimer of high molecular weight $(260 \mathrm{kDa})$ consisting of 16-18 monomers of $15.3 \mathrm{kDa}$. Metal content, EXAFS data and DFT calculations support the presence of a Mo-2S-[2Fe-2S]-2S-Mo cluster never reported before. MorP encoding gene is located downstream of a sensor (mor $S)$ and a regulator gene ( $m o r R)$, encoding a two-component system. Increase of both gene expression and protein production was observed when cells were cultured in the presence of $45 \mu \mathrm{M}$. In the same growth conditions the $\operatorname{morR}$ gene expression was also up-regulated. MorR was heterologously expressed in E. coli and is currently being characterized.

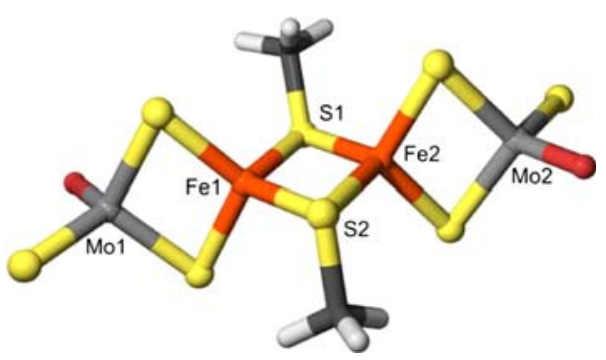

Acknowledgements: We thank Fundação para a Ciência e a Tecnologia for financial support (POCI/QUI/55350/2004) and Ciência 2007

\section{References}

1. Rivas MG, Carepo MSP et al (2009) Biochemistry 48:873-882

\section{K-13}

\section{Regulation of interdomain electron transfer} in the output state of nitric oxide synthase Changjian Feng1, J. Guy Guillemette ${ }^{2}$, Martin Kirk ${ }^{3}$

${ }^{1}$ College of Pharmacy, University of New Mexico, Albuquerque, NM 87131, USA.

${ }^{2}$ Department of Chemistry, University of Waterloo, Waterloo, ON N2L 3G1, Canada.

${ }^{3}$ Department of Chemistry and Chemical Biology, University of New Mexico, Albuquerque, NM 87131, USA. cfeng@salud.unm.edu NO synthesis by NO synthase (NOS) is tightly regulated by catalytically relevant interdomain electron transfer (IET) processes. The 
NOS output state is a complex between the FMN and heme domains, and it facilitates the FMN-heme IET that delivers electron(s) required for $\mathrm{O}_{2}$ activation in the catalytic heme domain. Very recently, we have designed a $\mathrm{CO}$ photolysis method to directly determine the FMN-heme IET kinetics in a truncated NOS construct [1,2], which is designed to favor observation of the NOS output state. We have further extended our studies to full length nNOS and iNOS enzymes [3]. Our results demonstrate that calmodulin modulates formation of the NOS output state through facilitating interdomain FMN/heme interactions. This work strongly supports a tethered shuttle model that involves the swinging of the FMN domain. The first direct spectroscopic investigation of the interdomain FMN/heme interactions will also be presented.

\section{References}

1. Feng CJ, Tollin G, Holliday MA, Thomas C, Salerno JC, Enemark JH, Ghosh DK (2006) Biochemistry 45:6354-6362

2. Feng CJ, Thomas C, Holliday MA, Tollin G, Salerno JC, Ghosh DK, Enemark JH (2006) J Am Chem Soc 128:3808-3811

3. Feng CJ, Tollin G, Hazzard JT, Nahm NJ, Guillemette JG, Salerno JC, Ghosh DK (2007) J Am Chem Soc 129:5621-5629

\section{K-14}

\section{Type zero copper proteins}

Kyle M. Lancaster ${ }^{1}$, Serena DeBeer George ${ }^{2}$, Keiko Yokoyama ${ }^{1}$, John H. Richards ${ }^{1}$, Harry B. Gray ${ }^{1}$

${ }^{1}$ Division of Chemistry and Chemical Engineering, California

Institute of Technology, Pasadena, CA 91125, USA.

${ }^{2}$ Stanford Synchrotron Radiation Laboratory, Menlo Park,

CA 94025, USA. Km104747@ caltech.edu

The chemical and spectroscopic properties of proteinaceous metal sites have long been known to exhibit dramatic sensitivity to inner and outer sphere coordination. In attempting to access highpotential, hard ligand-based copper sites we have observed [1] in certain mutants of Pseudomonas aeruginosa azurins a peculiar $\mathrm{Cu}$ (II) binding site displaying spectroscopic and chemical features atypical of mononuclear proteinaceous $\mathrm{Cu}(\mathrm{II})$. Specifically, these sites possess the low axial hyperfine splitting $\left(A_{\|} \sim 10 \mathrm{mK}\right)$ in their EPR spectra and elevated reduction potentials $\left(E^{\circ} \sim 300 \mathrm{mV}\right.$ vs. NHE) of type 1 copper while lacking the associated "blue copper" charge transfer absorption. We have elected to call these non-natural sites "type 0 ," in keeping with their resemblance to type 1. X-ray crystallography of a variety of "type zero" proteins reveals their $\mathrm{Cu}(\mathrm{II})$ to be distorted tetrahedra with a unprecedented short $\mathrm{Cu}$ (II) to backbone carbonyl bond distances $(2.35-2.55 \AA)$. XANES reveal substantial $4 p$ orbital mixing into the $3 d$ ground state evidenced by gain of intensity at $8979 \mathrm{eV}$ relative to an analogous "type 2" copper site. This feature appears to be correlated to $\mathrm{Cu}$ (II) to Gly45 carbonyl bond distance. The low axial hyperfine splitting suggests a low-lying excited state, supported by observation of paramagnetically shifted resonances in the ${ }^{1} \mathrm{H}$ NMR spectrum. Electron transfer (ET) studies conducted via SAMmodified $\mathrm{Au}$ electrochemistry indicate decreased reorganization energy in "type zero" sites. We are currently conducting EXAFS analysis of oxidized and reduced sites to probe ET functionality, and have initiated collaborations to measure ET rates via pulse radiolysis and to elucidate the ground state via W-band ENDOR and ELDOR.

\section{Reference}

1. Lancaster KM, Yokoyama K, Richards JH, Winkler JR, Gray HB (2009) Inorg Chem 48:1278-1280

\section{K-15}

Four-electron reduction of dioxygen by a multicopper oxidase, CueO, and roles of Asp112 and Glu506 located adjacent to the trinuclear copper center

Takeshi Sakurai $^{1}$, Kunishige Kataoka ${ }^{1}$, Ryosuke Sugiyama ${ }^{1}$, Shun Hirota ${ }^{2}$, Megumi Inoue ${ }^{1}$, Kanae Urata ${ }^{1}$, Yoichi Minagawa ${ }^{1}$, Daisuke Seo ${ }^{1}$

${ }^{1}$ Graduate School National Science and Technology, Kanazawa University, Kanazawa 920-1192, Japan.

${ }^{2}$ Graduate School of Materials Science, Nara Institute of Science and Technology, Ikoma, Nara 630-019, Japan.

ts0513@kenroku.kanazawau.ac.jp

The mechanism of the four-electron reduction of dioxygen by a multicopper oxidase, $\mathrm{CueO}$, was studied based on reactions of single and double mutants with Cys500, a type I Cu ligand, and the non-coordinating Asp112 and Glu506, which form hydrogen bonds with the trinuclear $\mathrm{Cu}$ center directly and indirectly via a water molecule. The reaction of Cys500Ser containing a vacant type $\mathrm{I} \mathrm{Cu}$ center produced the intermediate $\mathrm{I}$ in an EPR-silent peroxide-bound form. The formation of the intermediate $\mathrm{I}$, the peroxide bound form, from Cys500Ser/Asp112Asn was much slow and less due to a reduction in the affinity of the trinuclear $\mathrm{Cu}$ center for dioxygen. The state of the intermediate I was realized to be the resting form of Cys500Ser/Glu506Gln and Cys500Ser of the truncated mutant $\Delta \alpha 5-7 \mathrm{CueO}$, in which the 50 amino acids covering the substrate-binding site were removed [1]. Reactions of the recombinant $\mathrm{CueO}$ and Glu506Gln afforded the intermediate II, a fully oxidized form different from the resting one, with a very broad EPR signal, $g<2$, detectable only at cryogenic temperatures and unsaturated with high power microwaves. The lifetime of the intermediate II was prolonged by the mutation at Glu506 involved in the donation of protons. The structure of the intermediates I and II and the mechanism of the four-electron reduction of dioxygen driven by the non-coordinating acidic amino acids, Asp112 and Glu506, located adjacent to the trinuclear $\mathrm{Cu}$ center are discussed.

\section{References}

K. Kataoka et al., J. Mol. Biol., 373, 141-152 (2007).

\section{K-16}

Effects of nickel and $\mathrm{pH}$ on Helicobacter pylori NikR Yanjie Li, Deborah B. Zamble

Department of Chemistry, University of Toronto, Toronto M5S 3H6, Canada.yli@chem.utoronto.ca

Helicobacter pylori $(H$. pylori) is a microaerophilic pathogenic bacteria that resides in the human stomach, where it survives extreme acidic conditions and infects half of the world's population. Efficient colonization by $H$. pylori requires urease and hydrogenase enzymes, both of which utilize nickel as a cofactor. Cellular level of nickel in $H$. pylori is strictly maintained by a nickel-responsive transcription factor, HpNikR, which acts as a master regulator to control both activation of the urease genes and repression of a variety of other genes including its own. In addition to its role in nickel homeostasis, HpNikR has been implicated in the adaptive response of $H$. pylori to acidic environmental conditions. However, it is not clear whether the drastic decrease of external $\mathrm{pH}$ will affect the properties of HpNikR and how different genes are distinctively regulated by $\mathrm{HpNikR}$. To answer these questions, we used electrophoresis mobility shift assays and DNase footprinting assays, and discovered that the DNA-binding activity 
of $\mathrm{HpNikR}$ on several promoters is selectively controlled by $\mathrm{pH}$ and/or nickel, and the effect of $\mathrm{pH}$ on different promoters depends on the recognition sequence. In addition, several amino acids at the $\mathrm{N}$ terminus of HpNikR are critical for the specific responses of HpNikR. The physiological relevance of these in vitro results is currently being tested in vivo. Given the relatively low abundance of transcription factors in $H$. pylori, information about the effects of nickel and $\mathrm{pH}$ on HpNikR is important for understanding the multifaceted roles of HpNikR in fine-tuning the physiology of this organism.

\section{References}

1. Dunn BE, Cohen H, Blaser MJ (1997) Clin Microbiol Rev 10:720 2. van Vliet AH, Ernst FD, Kusters JG (2004) Trends Microbiol $12: 489$

3. Abraham LO, Li Y, Zamble DB (2006) J Inorg Biochem 100:1005

4. Li Y, Zamble DB (2009) Biochemistry (in press)

\section{K-17}

Analyses of the heme ligand-field anisotropy in a series of ferric low spin cytochrome $c$ mutants Giorgio Zoppellaro', Kara L. Bren ${ }^{2}$, Espen Harbitz ${ }^{1}$, Ravinder Kaur ${ }^{2}$, Amy A. Ensign², K. Kristoffer Andersson ${ }^{1}$ ${ }^{1}$ Department of Molecular Biosciences, University of Oslo, 0316 Oslo, Norway.

${ }^{2}$ Department of Chemistry, University of Rochester, Rochester, NY 14627-0216, USA

A series of cytochrome $c$ mutants derived from Pseudomonas aeruginosa ( $\mathrm{Pa} c$-551) and from the ammonia oxidizing bacterium Nitrosomonas europaea ( $\mathrm{Ne} c$-552) were over-expressed. Point mutations were induced in a key residue nearby the Met axial ligand (Asn64). The effects were probed by low-temperature electron paramagnetic resonance (EPR) and nuclear magnetic resonance $\left({ }^{1} \mathrm{H}\right.$ NMR) spectra analyses. Ne $c$-552 has a ferric low spin $(S=1 / 2)$ EPR signal characterized by large $g$ anisotropy and $g_{\max }$ resonance at 3.34 (see Fig. 1), while Pa $c-551$ ( $g_{\max }$ at 3.20$)$ exhibits electronic features consistent with very weak axial system. Correlation among the electronic fingerprints observed in the over-expressed proteins as well as in their mutants [1] shows the occurrence of a linear relation between axial strains (or $g_{\max }$ values) and averaged paramagnetic methyl shifts that stands irrespective of their specific Met dynamics [2].

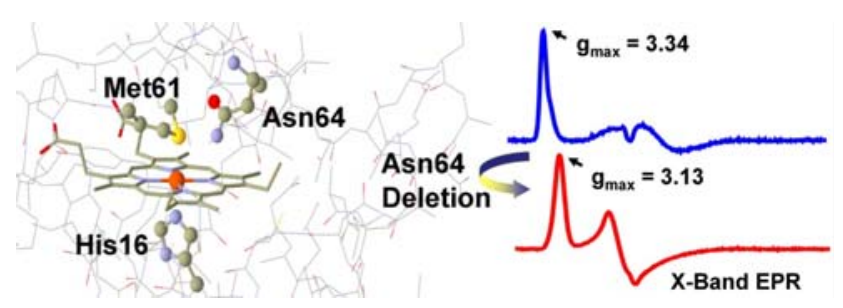

Fig. 1

\section{References}

1. Zoppellaro G, Harbitz E, Kaur R, Ensign AA, Bren KL, Andersson KK (2008) J Am Chem Soc 130:15348-15360

2. Zoppellaro G, Bren KL, Ensign AA, Harbitz E, Kaur R, Hersleth HP, Ryde U, Hederstedt L, Andersson KK Biopolymers (submitted)

\section{K-18}

Structure and catalytic mechanism of heme oxygenase Masaki Unno, Toshitaka Matsui, Masao Ikeda-Saito

${ }^{1}$ Institute of Multidisciplinary Research for Advanced Materials,

Tohoku University, Sendai 980-8577, Japan.

unno19@tagen.tohoku.ac.jp

Heme oxygenase $(\mathrm{HO})$ catalyzes $\mathrm{O}_{2}$-dependent regiospecific conversion of heme to biliverdin, $\mathrm{CO}$ and free $\mathrm{Fe}(\mathrm{II})$. Heme degradation by HO proceeds via a multistep mechanism, in which $\mathrm{HO}$ oxidation consumes a total of three molecules of $\mathrm{O}_{2}$ and seven electrons. The catalytic mechanism of $\mathrm{HO}$ includes several interesting aspects. First, $\mathrm{HO}$ is not a hemeprotein per se but utilizes heme as both a substrate and a cofactor. Second, the $\mathrm{CO}$ produced by $\mathrm{HO}$ does not appear to severely interfere with heme degradation. Third, the ferric hydroperoxo species $(\mathrm{Fe}-\mathrm{OOH})$ in $\mathrm{HO}$ self-hydroxylates the $\alpha$-meso-carbon of the porphyrin ring to generate $\alpha$-meso-hydroxyheme in HO. This is different from $\mathrm{P} 450$, peroxidases and catalases, where the $\mathrm{O}-\mathrm{O}$ bond of the $\mathrm{Fe}-\mathrm{OOH}$ species is heterolytically cleaved to generate an actively hydroxylating compound I, a ferryl $\left(\mathrm{Fe}^{4+}=\mathrm{O}\right)$ species coupled with a porphyrin $\pi$-cation radical. Fourth, heme cleavage by HO displays regioselectivity such that only the $\alpha$-meso-carbon is oxygenated. In order to understand the unique reaction mechanism, we have solved crystal structures of several catalytic intermediates of $\mathrm{HO}$ at high resolution. In this conference, we will report the relationships between structures of the catalytic intermediates and catalytic mechanism of HO.

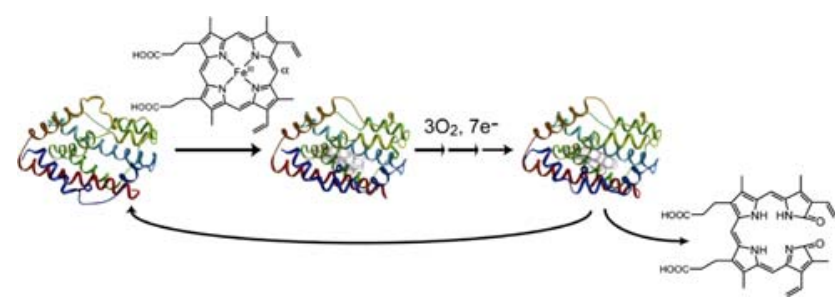

\section{K-19}

Spectroscopic and functional characterization of diiron monooxygenases involved in antibiotic biosynthesis Thomas M. Makris ${ }^{1}$, Mrinmoy Chakrabarti ${ }^{2}$, Eckard Münck ${ }^{2}$, John Lipscomb ${ }^{1}$

${ }^{1}$ Department of Biochemistry, Molecular Biology and Biophysics, University of Minnesota, Minneapolis, MN 55455, USA.

${ }^{2}$ Department of Chemistry, Carnegie Mellon University, Pittsburgh, PA 15213, USA.makri006@umn.edu

Natural product assembly lines often involve the use of tailoring enzymes which are critical for the resulting structure and bioactivity of the non-ribosomal peptide product. These include several types of oxygenases, which are involved in the biosynthesis of pharmaceutically important antimicrobial and cytostatic compounds. Notably, non-heme diiron monooxygenases are not known to catalyze these reactions. Analysis of the chloramphenicol biosynthesis gene cluster, chosen as a model system because of its structural simplicity, has now revealed the presence of two diiron monooxygenases, which we have recently recombinantly expressed and characterized. The first, a betahydroxylase, represents a novel diiron oxygenase motif which also appears to be utilized in the biosynthetic pathways of a number of pharmaceutical mainstays. We present optical, EPR, and Mössbauer spectra to establish the nature of the diiron cofactor. Also, analytical methods are used in concert with stopped flow kinetics to determine the substrate specificity, mechanism, and regulation. The second enzyme, an $\mathrm{N}$-oxygenase, belongs to a group that has been the subject of considerable debate regarding metal ion specificity and mechanism. We present the spectroscopic characterization of the enzyme, and utilizing metal titration and activity studies unambiguously 
assigns the diiron cofactor. Its role in chloramphenicol biosynthesis will be discussed.

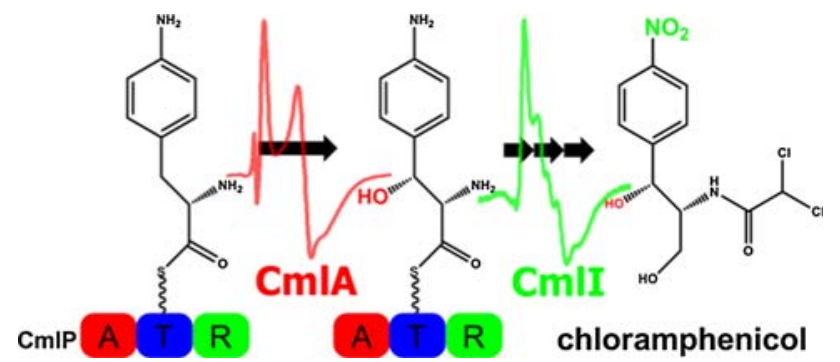

K-20

\section{Heme hydroperoxidase catalysis: the dynamic role} of distal side residues

Pietro Vidossich $^{1,2}$, Mercedes Alfonso-Prieto ${ }^{1,2}$, Carme Rovira $^{1,2,3}$

${ }^{1}$ Laboratori de Simulació Computacional i Modelització, Parc Científic de Barcelona, Josep Samitier 1-5, 08028 Barcelona, Spain. ${ }^{2}$ Institut de Química Teòrica i Computacional, Barcelona, Spain. ${ }^{3}$ Institució Catalana de Recerca i Estudis Avançats, Passeig Lluís Companys, 23, 08018 Barcelona, Spain. pvidossich@pcb.ub.es Heme enzymes catalyze a wide range of chemical reactions and research aimed to grasp the origin of this functional diversity is an extremely active field. We present results from modeling studies of peroxidase [1], catalase [2,3] and catalase-peroxidase [4] from which the role played by distal side residues clearly emerges. By means of hybrid QM/MM MD simulations we have investigated the atomic and electronic reorganization taking place during (1) the formation of the high valent iron-oxo species known as Compound I (Cpd I) in Horseradish peroxidase from reaction with $\mathrm{H}_{2} \mathrm{O}_{2}$, (2) the reduction of Cpd I by $\mathrm{H}_{2} \mathrm{O}_{2}$ in $H$. pylori heme-b catalase and $P$. vitale heme-d catalase. Neither of the two reactions proceed in a single step, and reorganization of the intervening species is needed between successive steps, revealing the motion of catalytic residues.

Furthermore, we have characterized the electronic structure of Burkholderia pseudomallei catalase-peroxidase (KatG) Cpd I, showing that the radical character of $\mathrm{Cpd} \mathrm{I}$ is affected by the protonation state of the KatG specific Met-Tyr-Trp covalent adduct present at the active site.

\section{References}

1. Derat E, Shaik S, Rovira C, Vidossich P, Alfonso-Prieto M (2007) J Am Chem Soc 129:6346-6347

2. Alfonso-Prieto M, Borovik A, Carpena X, Murshudov G, MelikAdamyan W, Fita I, Rovira C, Loewen PC (2007) J Am Chem Soc 129:4193-4205

3. Alfonso-Prieto M, Biarnés X, Vidossich P, Rovira C (Submitted) 4. Vidossich P, Alfonso-Prieto M, Carpena X, Loewen PC, Fita I, Rovira C (2007) J Am Chem Soc 129:13436-13446

\section{K-21}

\section{Functional and mechanistic studies on human}

\section{2-oxoglutarate dependent oxygenases}

\section{Christopher J. Schofield}

The Department of Chemistry and the Oxford Centre for Integrative Systems Biology, Mansfield Road, Oxford OX1 5JJ, UK.

Christopher.schofield@chem.ox.ac.uk

2-Oxoglutarate and $\mathrm{Fe}$ (II)-dependent oxygenases have long been known to catalyze the post-translational modification of collagen. More recent studies have shown that they play a central role in hypoxic sensing via the hydroxylation of the hypoxia inducible transcription factor (HIF). A set of oxygenses catalyse HIF prolyl hydroxylation, which signals for HIF degradation. HIF asparaginyl hydroxylation, as catalyzed by FIH, reduces the interaction of HIF with the p300 transcriptional coactivator. FIH is closely related to an extended family of histone methyl lysine-residue demethylases. The lecture will describe new roles for human $20 \mathrm{OG}$ oxygenases and discuss how the structures and mechanisms of these enzymes are related to their physiological roles.

\section{References}

1. Chowdhury R, Hardy A, Schofield CJ (2008) The human oxygen sensing machinery and its manipulation. Chem Soc Rev 37:1308-1319 2. Loenarz C, Schofield CJ (2008) Expanding chemical biology of 2-oxoglutarate oxygenases. Nat Chem Biol 4:152-156

3. T. Gerken et al (2007) The obesity-associated FTO gene encodes a 2-oxoglutarate-dependent nucleic acid demethylase. Science 318:1469-1472

4. Ng SS et al (2007) Crystal structures of histone demethylase JMJD2A reveal basis for substrate specificity. Nature 448:87-91

\section{K-22}

Catalytic mechanism of nitrile hydratase proposed by time-resolved X-ray crystallography using a novel substrate, tert-butylisonitrile

Masafumi Odaka ${ }^{1}$, Koichi Hashimoto ${ }^{1}$, Hiroyuki Suzuki ${ }^{2}$, Kayoko Taniguchi ${ }^{3}$, Takumi Noguchi ${ }^{2}$, Masafumi Yohda ${ }^{1}$

${ }^{1}$ Department of Biotechnol, Graduate School of Engineering, Tokyo University of Agriculture and Technology.

${ }^{2}$ Institute of Materials Science, Univerity of Tsukuba.

${ }^{3}$ Eco-Soft Material Research Units, RIKEN. modaka@cc.tuat.ac.jp Nitrile hydratases (NHases) have an unusual iron or cobalt catalytic center with two oxidized cysteine ligands, cysteine-sulfinic acid and cysteine-sulfenic acid (Cys-SOH), catalyzing the hydration of nitriles to the amides. Recently, we found that the NHase of Rhodococcus erythropolis $\mathrm{N} 771$ exhibited an additional catalytic activity, converting tertbutylisonitrile $(t \mathrm{BuNC})$ to tert-butylamine [1]. Taking advantage of the slow reactivity of $t \mathrm{BuNC}$ and the photoreactivity of nitrosylated NHase, we present the first structural evidence for the catalytic mechanism of NHase with time-resolved X-ray crystallography [2]. Using ATR-FTIR spectroscopy, the product from the isonitrile carbon was identified as a $\mathrm{CO}$ molecule. Crystals of nitrosylated-inactive NHase were soaked with $t$ BuNC. The catalytic reaction was initiated by photo-induced denitrosylation and stopped by flash-cooling. $t$ BuNC was first trapped at the hydrophobic pocket above the $\mathrm{Fe}$ center and then coordinated to the $\mathrm{Fe}$ ion at $120 \mathrm{~min}$. At $440 \mathrm{~min}$, the electron density of $t$ BuNC was significantly altered, and a new electron density was observed near the isonitrile carbon as well as the sulfenate oxygen of $\alpha$ Cys114. These results demonstrate that the substrate was coordinated to the iron and attacked by a solvent molecule activated by $\alpha \mathrm{Cys} 114-\mathrm{SOH}$.

\section{References}

1. Taniguchi K, Murata K, Murakami Y, Takahashi S, Nakamura T, Hashimoto K, Koshino K, Dohmae N, Yohda M, Hirose T, Maeda M, Odaka M (2008) J Bioeng Biosci 106:174-179

2. Hashimoto K, Suzuki H, Taniguchi K, Noguchi T, Yohda M, Odaka M (2008) J Biol Chem 283:36617-36623

\section{K-23}

Role of sulfenate coordination in nitrile hydratase through a model complex chemistry

Tomohiro Ozawa, Takuma Yano, Yasuhiro Funahashi, Hideki Masuda 
Department of Frontier Materials, Graduate School of Engineering, Nagoya Institute of Technology, Nagoya 466-8555, Japan. ozawa.tomohiro@nitech.ac.jp

Nitrile hydratase (NHase), which hydrates a nitrile compound to the corresponding amine, has an $\mathrm{Fe}$ (III) or a $\mathrm{Co}$ (III) center in the active site. The coordinated three sulfurs were in a different oxidation state (thiolate, sulfenate, and sulfinate), which is an essential factor for the NHase activation, because over oxidation to sulfinates caused a deactivation [1]. Many studies have been performed to understand the reaction mechanism on the unique structure, but it has not been clarified yet. We prepared model complexes and investigated the role of the unstable sulfenate coordination.

Crystal structure of a Co(III) complex with two sulfenate donors [2] is shown in Fig. 1. The sulfenate groups easily encountered an overoxidation in DMF at RT, while did not in methanol, indicating that the interaction contributed to the stabilization of the sulfenate state. The $\mathrm{S}-\mathrm{O}$ stretching frequency depended on accepter numbers (ANs) of solvent that is an index of the electrophilicity. Thus, the sulfenate coordination showing a nucleophlicity may contribute to the enzymatic reaction of NHase.

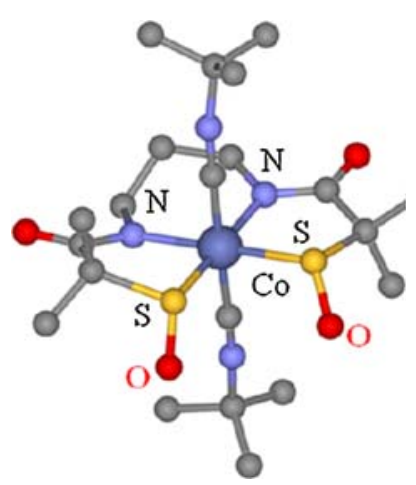

Fig. 1 Crystal structure of an NHase model complex with two sulfenates

\section{References}

1. Tsujimura M, Odaka M, Nakayama H, Dohmae N, Koshino H, Asami T, Hoshino M, Takio K, Yoshida S, Maeda M, Endo I (2003) J Am Chem Soc 125:11532-11538

2. Yano T, Wasada-Tsutsui Y, Arii H, Yamaguchi S, Funahashi Y, Ozawa T, Masuda H (2007) Inorg Chem, 46(24):10345-10353

\section{K-24}

Wheat $\mathrm{Zn}_{4} \beta_{\mathrm{E}^{-}} E_{\mathrm{c}}-1$ : the first 3D structure of a plant metallothionein

\section{Eva Freisinger}

Inorganic Chemistry, University of Zurich, 8057 Zurich, Switzerland. freisinger@aci.uzh.ch

Triticum aestivum (bread wheat) $E_{\mathrm{c}}-1$ was the first metallothionein discovered in higher plants [1]. Although isolation of the protein from wheat germs dates back more than 25 years, so far knowledge about its metal-binding abilities and spectroscopic properties was surprisingly limited. In its own way, it is only just and equitable that wheat $E_{\mathrm{c}}-1$ became now as the first plant metallothionein, whose threedimensional structure has been solved [2]. The metal ions in $\mathrm{Zn}_{6} E_{\mathrm{c}}-1$ are arranged in two domains, the N-terminal $\mathrm{Zn}_{2} \gamma-E_{\mathrm{c}}-1$ and the C-terminal $\mathrm{Zn}_{4} \beta_{\mathrm{E}}-E_{\mathrm{c}}-1$ domain $[3,4]$, the solution structure of the latter being described here as determined by NMR spectroscopy.
The $\beta_{\mathrm{E}}$-domain, or extended $\beta$-domain, features a unique and unprecedented arrangement of a mononuclear $\mathrm{ZnCys}_{2} \mathrm{His}_{2}$ site and a $\mathrm{Zn}_{3} \mathrm{Cys}_{9}$ cluster similar to the ones observed in vertebrate MTs. In addition, the metal ion coordination properties of both domains will be discussed, with a special emphasis on the presence of domain interactions, cooperative effects and the influence of the redox state of the solution.

Financial support from the Swiss National Science Foundation is gratefully acknowledged: SNF grant 20-113728/1 and SNFFörderungsprofessur PP002-119106/1.

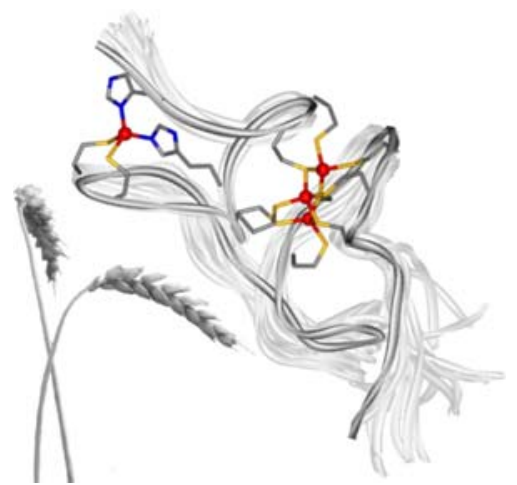

References

1. Hanley-Bowdoin L, Lane BG (1983) Eur J Biochem 135:9-15

2. Peroza EA, Schmucki R, Güntert P, Freisinger E, Zerbe O (2009) $\mathrm{J}$ Mol Biol (in press)

3. Peroza EA, Freisinger E (2007) J Biol Inorg Chem 12:377-391

4. Peroza EA, Al Kaabi A, Meyer-Klaucke W, Wellenreuther G, Freisinger E (2009) J Inorg Biochem 103:342-353

\section{K-25}

The properties of the multi-component enzymes alcohol and aldehyde dehydrogenases-fixing Gluconacetobacter diazotrophicus

S. Gómez-Manzo ${ }^{1}$, A. Solano-Peralta ${ }^{1}$, J.E. Escamilla-Marván ${ }^{2}$, P.M.H. Kroneck ${ }^{3}$, M.E. Sosa-Torres ${ }^{1}$

${ }^{1}$ Departamento de Química Inorgánica y Nuclear, Facultad de Química,

${ }^{2}$ Instituto de Fisiología Celular, Universidad Nacional Autónoma de México, Ciudad Universitaria 04510 México, D.F., Mexico.

${ }^{3}$ Fachbereich Biologie, Universität Kostanz, 78457 Konstanz, Germany.mest@servidor.unam.mx

Gluconacetobacter diazotrophicus is a dinitrogen-fixing endophyte originally isolated from sugar cane. These microorganisms are able to oxidize ethanol to acetic acid which is catalyzed by two membraneous enzymes complexes, alcohol dehydrogenase (ADH) and aldehyde dehydrogenase (ALDH). Both enzymes are quino-hemoproteins and carry PQQ and cytochrome $c$ as prosthetic groups [1]. In general, multi-heme proteins are important constituents of bacterial electron transfer chains and are involved in energy conservation [2]. In dinitrogen-fixing bacteria, such as Ga. diazotrophicus, both ADH and ALDH may also help to protect the dioxygen-sensitive iron-sulfur enzyme nitrogenase [3]. Several alcohol and aldehyde dehydrogenase complexes have been isolated from the membrane fraction of acetic acid bacteria and purified to homogeneity [4]. Their three-dimensional structures and prosthetic groups are of great interest for applications in biotechnology. Here, we report on the molecular and catalytic properties of the ADH and ALDH complexes isolated and purified from $\mathrm{Ga}$. diazotrophicus. The $\mathrm{ADH}$ complex is a heterodimer 
( $\alpha 72 \mathrm{kDa}, \beta 44 \mathrm{kDa}$ ) and contains one PQQ and four $c$-type cytochrome centers. The ALDH complex exhibits also a heterodimeric architecture $(\alpha 80 \mathrm{kDa}, \beta 50 \mathrm{kDa})$ and appears to harbor one PQQ, three $\boldsymbol{c}$-type cytochrome centers and one $\boldsymbol{b}$-type cytochrome. Furthermore, a molybdopterin site has been discovered most recently as an additional prosthetic group of this ALDH complex.

\section{References}

1. Gómez-Manzo S, Contreras-Zentella M, González-Valdés A, SosaTorres ME, Arreguín-Espinoza R, Escamilla-Marván JE (2008) Int J Food Microbiol 125:71-78

2. Matias PM, Pereira IAC, Soares CM, Carrondo MA (2005) Prog Biophys Mol Biol 89:292-329

3. Flores-Encarnación M, Contreras-Zentella M, Soto-Urzua L, Aguilar LR, Baca BE, Escamilla-Marván JE (1999) J Bacteriol 181:6987-6999

4. Matsushita K, Yakushi T, Takaki Y, Toyama H, Adachi O (1995) J

Bacteriol 177:6552-6559

\section{K-26}

Interaction of pseudopeptidic disulfides and disulfideoxides towards metal cations: relation to biological

\section{systems}

\section{Isabelle Artaud}

Laboratoire de Chimie et Biochimie, Pharmacologique et

Toxicologique, UMR8601 CNRS Université Paris Descartes, 45 rue des Sts Pères, 75270, Paris Cedex 06, France.

Isabelle.artaud@parisdescartes.fr

All the sulfur-oxidized species derived from thiols or disulfides have been clearly identified in biological systems and are now supposed to play a key role in enzyme activity or as signaling agents. For example, the reduction of sulfinates involves the intermediate formation of an interprotein thiosulfinate [1]. Disulfides are found in Hepcidin, a 25 aa peptide responsible for the regulatory of iron homeostasis [2]. This system is supposed to bind iron. The only systems which contain oxidized cysteines, including the highly unstable sulfenate, ligated to a metal center are nitrile hydratases [3] and thiocyanate hydrolases. The processes of oxidation of these cysteines are however not clearly elucidated.

The chemical approach using cyclic pseudopeptidic thiosulfinates or thiosulfonates $[4,5]$, as well as cyclic or acyclic pseudopeptidic bisdisulfides allows to propose some new hypotheses to explain the formation of the nitrile hydratase metal active site and the possible reversible metallation of hepcidin.

\section{References}

1. Vivancos AP, Castillo EA, Biteau B, Nicot C, Ayte J, Toledano MB, Hidalgo E (2005) Proc Natl Acad Sci USA (2005) 102:8875

2. Hunter HN, Fultan DB, Ganz T, Vogel HJ (2002) J Biol Chem 277:37597-37603

3. Miyanaga A, Fushinobu S, Ito K, Wakagi T (2001) Biochem Biophys Res Commun 288:1169-1174
4. Galardon E, Bourlès E, Artaud I, Daran J.-C., Roussel P, Tomas A (2007) Inorg Chem 46:4515-4522

5. Bourlès E, Alves de Sousa R, Galardon E, Giorgi M, Artaud I (2005) Angew Chem Int Ed 44:6162-6165

\section{K-27}

Towards a reaction mechanism for the sulfur oxygenase reductase of the thermoacidophilic archaeon Acidianus ambivalens

Arnulf Kletzin ${ }^{1}$, Andreas Veith, Kerstin Seyfart, Tim Urich ${ }^{1,2}$, Miguel Teixeira ${ }^{3}$, Carlos Frazão ${ }^{3}$

${ }^{1}$ Institute of Microbiology and Genetics, Darmstadt University of Technology, Schnittspahnstr. 10, 64287 Darmstadt, Germany.

${ }^{2}$ Present Address: Department of Genetics in Ecology,

University of Vienna, Vienna, Austria. ${ }^{3} \mathrm{ITQB}$, Av. da República (EAN), 2781-901 Oeiras, Portugal. Kletzin@bio.tu-darmstadt.de The soluble sulfur oxygenase reductase (SOR) is the initial enzyme in sulfur oxidation by the archaeon Acidianus ambivalens. The globular, hollow 24-subunit enzyme with 432-point group symmetry [1] catalyzes the $\mathrm{O}_{2}$-dependent sulfur disproportionation to sulfite and $\mathrm{H}_{2} \mathrm{~S}$. The active sites in each subunit comprise a mononuclear low-potential non-heme iron and a cysteine persulfide in a spacious pocket [2]. Two consecutive pores provide substrate access to the active sites. The pores in the outer shell formed by chimney-like protrusions at the fourfold symmetry axis have an apolar interior suggesting a substrate entry pathway [1]. Pore opening lead to an increase in activity [3]. The second pore formed by Met and $\mathrm{Val}$ residues provides entrance to the active site cavity. Mutagenesis resulted in a decrease of enzyme activity unless the pores remain intact. Binding of $\mathrm{Zn}^{2+}$ ions blocks the putative pathway of product exit at the threefold symmetry axis. A hydrogen-bonding network in the second coordination sphere surrounding the metal ion presumably contributes to the low reduction potential [1]. Mutagenesis of surrounding residues reduced but did not fully abolish SOR activity [3]. Derivatives with the $\mathrm{Fe}^{3+}$ ion exchanged for $\mathrm{Co}^{2+}, \mathrm{Mn}^{2+}, \mathrm{Ni}^{2+}$, and $\mathrm{Ga}^{3+}$ resulted in active enzyme suggesting that a valence change does not occur during catalysis and that $\mathrm{O}_{2}$ is activated by the substrate and not by the metal [3]. We propose a reaction mechanism involving a hydroxyl-catalyzed nucleophilic cleavage of cysteine-bound polysulfide as the initial step. The resulting polysulfenic acid should act as a strong reductant for activation of $\mathrm{O}_{2}$ and formation of polysulfonic acid as the primary product.

\section{References}

1. Urich T, Gomes CM, Kletzin A, Frazao C (2006) Science 311:9961000

2. Urich T, Kroke A, Bauer C, Seyfarth K, Reuff M, Kletzin A (2005) FEMS Microbiol Lett 48:171-176

3. Veith A, Seyfarth K, Urich T, Protze J, Teixeira M, Frazão C (unpublished) 